\title{
Allergies in Families of Moscow (Russia) Newborns: Suspected Cases within Three Generations Framework
}

\author{
Marina Treneva ${ }^{1, *}$, Alexander Pampura ${ }^{1}$, Daniel Munblit ${ }^{2}$ \\ ${ }^{1}$ Allergy and Clinical Immunology Unit, Research and Clinical Institute for Pediatrics at the Pirogov Russian National Research \\ Medical University, Moscow, Russia \\ ${ }^{2}$ Department of Paediatrics, Imperial College London, London, United Kingdom \\ *Corresponding author: trenevamarina@mail.ru
}

Received March 30, 2015; Revised April 12, 2015; Accepted April 21, 2015

\begin{abstract}
Background: Allergy prevalence in parents of newborns is an indicator of allergic diseases in children, which is split upon parent's gender and allergic conditions. Objective: to find out gender patterns within a larger framework of allergies in grandparents, aunts, uncles of newborns in maternal and paternal families. Methods: In 201113 visits to the Postnatal Department of Moscow No.1 Maternity Hospital were carried out. All women delivered within the preceding 48 hours were interviewed. Mothers provided us with information on relatives of a newborn in regards to clinical manifestations of food allergies, atopic dermatitis, eczema, urticaria, angioedema, allergic rhinitis, allergic conjunctivitis, asthma and episodes of drug allergy. Information on 3534 relatives from 393 newborns was collected. Results: The rate of suspected allergic conditions in grandfathers is the same in both maternal and paternal families (4,35\% 95\%CI 1,99-6,70 and 4,62\% 95\%CI 2,12-7,12 respectively), but in grandmothers and aunts the rate of suspected allergic incidence is increased in maternal family $(11,54 \% 95 \% \mathrm{CI}$ 8,58-14,50) over paternal $(6,80 \%$ 95\%CI 4,46-9,14). The cumulative rate of suspected allergies in grandfathers+fathers+uncles (i.e. in male relatives of two preceding the newborns generations) is significantly lower $(8,57 \%$ 95\%CI 7,03-10,11) than in grandmothers+mothers+aunts (i.e. in female relatives of two preceding the newborns generations) $(13,28 \%$ 95\%CI 11,44-15,13) $(\mathrm{p}<.05)$. Conclusion: There are clear gender differences with increased prevalence of suspected allergic conditions in female relatives of two preceding the newborn generations compared with male relatives. There is a tendency to increased suspected allergic manifestations among women of maternal relatives in comparison to paternal relatives.
\end{abstract}

Keywords: allergy, newborns, relatives, parents, grandparents, cousins, siblings, uncles, aunts, prevalence

Cite This Article: Marina Treneva, Alexander Pampura, and Daniel Munblit, "Allergies in Families of Moscow (Russia) Newborns: Suspected Cases within Three Generations Framework." American Journal of Public Health Research, vol. 3, no. 3 (2015): 95-99. doi: 10.12691/ajphr-3-3-3.

\section{Introduction}

Knowledge of allergic conditions in the relatives of newborns is useful for the primary prevention of allergic diseases in children. Research has most commonly focused on the parents and siblings of newborns and varied uncertain upon parent's gender and allergic conditions. Work of Wen et al [1] demonstrated an interesting role for the impact of paternal allergy on the risks of their offspring developing allergies. In $\mathrm{AD}$, the authors found children of mothers diagnosed with $\mathrm{AD}$ had a slightly higher risk of $\mathrm{AD}$ development compared with children of fathers diagnosed with $\mathrm{AD}$ (adjusted $\mathrm{OR}[95 \% \mathrm{CI}]=4.4$ [0.9-17.0] and 3.1 [0,4-13,1], respectively). However, an opposite result was found with respiratory allergic diseases (RAD) in parents (adjusted OR [95\% CI] $=1.4$ [0.6-2.9] and 2.2 [1,1-4,4] for mothers and fathers, respectively). Furthermore, 2 year old children whose fathers have had RAD become more at risk of developing $\mathrm{AD}$ than same age children whose mothers have been diagnosed with RAD (adjusted OR [95\% CI] $=2.2$ [1.14.4] and 1.4 [0.6-2.9], respectively).

We also know that there are age and sex differences in asthma prevalence, as noted by Kynyk et al [2], Lødrup Carlsen et al [3], where the incidence is higher in males before puberty. Puberty decreases male asthma prevalence and in contrast increases asthma prevalence in females. The latter may influence the predominant prevalence in mothers' allergy in comparison to fathers' in a family history of allergic diseases.

It appears that there are different risks of developing specific allergies conferred to a child dependent upon which parent is diagnosed allergic, as noted by Arshad et al [4]. This highlights the need for a study investigating the prevalence of allergy across wider family relatives. A cross- and same-generational framework should be used to find out the patterns conferred to offspring of developing allergies. Such framework should include grandparents and relatives of other degrees of kinship: uncles and aunts, siblings and cousins of newborns. According to our knowledge, there is no information on the prevalence of allergies in these relatives. Today, because of a longer- 
living population, we have the opportunity to study the incidence of allergies in cross-generational families.

Therefore, the aim of our study was to find out gender patterns within a large framework of allergies in grandparents, aunts, uncles of newborns in maternal and paternal families, as well as in parents, siblings and cousins of newborns.

\section{Methods}

The study has been approved by the medical ethics committee of the Research and Clinical Institute for Pediatrics at the Pirogov Russian National Research Medical University (protocol № 1-MS/11 18.01.2011).

13 visits, over a 2 month period (Oct - Dec 2011) were carried out at the postnatal department of Moscow No.1 maternity hospital. The admitted women were from Moscow metropolitan area. At each visit, all women who were admitted to the postnatal department delivered within the previous 48 hours were interviewed about their newborn's relatives' family history with respect to allergy. Interviews were carried out by an allergy specialist.

Clinical allergy information was obtained on the following relatives of the newborn child: grandmothers and grandfathers, aunts and uncles, sisters and brothers, male and female cousins. Stepbrothers or stepsisters were enrolled in the study because of shared blood affinity with a newborn. However, allergy history from newborn's parents step brothers or step sisters were not included in the study. Relatives with no information on their medical history were not included in the statistical analysis.

Mothers provided the study with information on herself and the father of a child as well as about each of the mentioned above relatives on clinical manifestations of food allergies, atopic dermatitis, eczema, urticaria, angioedema, allergic rhinitis, allergic conjunctivitis, asthma and episodes of drug allergy.

\subsection{Statistical Analysis}

The prevalence of allergy manifestations in newborns' relatives was calculated with 95\% confidence interval (95\% CI). A p-value less than 0.05 considered significant. «IBM SPSS Statistics Version 20» was used for data analysis.

\section{Results}

We obtained information on 3534 relatives from 393 newborns (see Table 1). Table 1 shows the breakdown of relatives and the absolute number of those relatives that have reported suspected allergies (food allergies, atopic dermatitis, eczema, urticaria, angioedema, allergic rhinitis, allergic conjunctivitis, asthma and episodes of drug allergy).

From the population studied, we found that 442 of 3534 relatives had a suspected allergic condition (12,51\% 95\%CI 11,35-13,67). Of these, almost one third of the relatives were accounted for by parents (specifically 98 mothers and 63 fathers). Maternal grandparents accounted for 63 allergic relatives, whilst paternal grandparents accounted for 44 allergic relatives. Same generation relatives (sisters, brothers, cousins) comprised 115 of the total number of relatives with allergic conditions.

We found a statistically significant gender difference in the incidence of suspected allergic conditions between two generations preceding the newborn irrespective of maternal or paternal lineage. The cumulative rate of suspected allergies in grandfathers, fathers and uncles (i.e. in male relatives of two preceding the newborns generations) is significantly lower $(8,57 \%$ 95\%CI 7,03$10,11)$ than in grandmothers, mohters and aunts (i.e. in female relatives of two preceding the newborns generations) $(13,28 \% 95 \%$ CI 11,44-15,13) ( $<$. .05). This gender difference was also noted when we looked at cumulative rates of suspected allergic conditions in all male relatives of newborns $10,17 \% \quad(8,71 \% \ldots 11,63 \%)$ versus $12,70 \%(11,12 \% \ldots 14,28 \%)$ in all female relatives.

The rate of suspected allergic conditions in grandfathers is the same in both maternal and paternal families (4,35\% 95\%CI 1,99-6,70 and 4,62\% 95\%CI 2,12-7,12 respectively), but in grandmothers and aunts the rate of suspected allergic incidence is increased in maternal family $(11,54 \% 95 \%$ CI $8,58-14,50)$ over paternal $(6,80 \%$ 95\%CI 4,46-9,14).

We found statistically significant differences in the incidence of suspected allergies between the different generations: grandparents prevalence is $7,04 \%$ (5,64\%...8,44\%); parents, aunts and uncles - 14,94\% (13,00\%...16,91\%); sisters/brothers and cousins - 21,18\% $(17,43 \% \ldots 24,92)$.

Relatives' kinships in the total number of suspected allergy manifestations are available in percentage (see Figure 1). This 'family' breakdown of allergic relatives covers those 442 of them who are known to have ever had any allergic manifestations. It should be noted that the number of maternal relatives is comparable to those of paternal ( $\mathrm{n}=1122$ and $\mathrm{n}=1083$, respectively).

\section{Discussion}

Considering that each visit to the postnatal department of the maternity hospital covered all mothers delivered in the preceding 48 hours, the results obtained could be linked with certain likelihood to population of city metropolitan area.

The females of any generation are seemed to be more capacious to allergy, whilst the male relatives of any generation are more rigid regardless to paternal or maternal family. The cumulative rate of allergic conditions in male relatives of two generations preceding the newborn is $8,57 \%$, almost half the cumulative allergic incidence in the same generations of female relatives $(13,28 \%)$. This gender difference, which is obvious in two preceding the newborns generations, could be seen in a case of three generations, where the third one is a generation of newborns (siblings and cousins). In this case the cumulative allergic incidence rate between all male relatives of newborns is $10,17 \%(95 \% \mathrm{CI} 8,71-11,63)$ and all female relatives $12,70 \%$ (95\%CI 11,12-14,28), suggesting that allergies may be more prevalence in females than in males.

We found an interesting fact that the incidence of allergic disease in grandfathers is nearly the same compared in maternal and paternal families (see Table 1). 
This might suggest that the incidence of allergy amongst grandfathers is more constant. Moreover, the allergies in grandfathers have a diagnostic specificity 94-97\%, which is the highest among all relatives' kinships, as noted by Treneva [5]. It could be useful for a primary prevention group setting with high specificity.

The highest frequency of allergies is among siblings of the newborns-2011 cohort. We found that the percentage of allergic sisters and brothers (30,0\% and 31,67\%, respectively) exceeded those for allergic mothers and fathers $(24,94 \%$ and $16,03 \%$, respectively). Prevalence dissonance is revealed in mixed data array regardless to any allergic form and corresponds to the results of Kynyk et al [2], Lødrup Carlsen et al [3] on respiratory allergies in males. The latter is of a higher prevalence before puberty. The same tendency has been shown for food allergy by Kanny et al [6]. Up to the age of 15, food allergy prevalence dominates in boys compared with girls. However, between the ages of 15-60 years, food allergy prevalence is higher in women. It is quite likely that age and sex dissonance in food and respiratory allergy manifestations prevalence is applicable to a wider range of allergic clinical manifestations, as could be seen in our results.

Female allergies gave an opposite result to those of Kynyk et al [2], Kanny et al [6]. We see a higher prevalence of allergies in sisters of newborns in compare to mothers of newborns.

Table 1. Suspected allergy manifestation prevalence in relatives of Moscow newborns-2011

\begin{tabular}{|c|c|c|c|c|}
\hline Relative kinships of newborns & $\mathrm{N}$ & $\begin{array}{c}\text { No. } \\
\text { of relatives with allergy } \\
\text { manifestations }\end{array}$ & $\begin{array}{l}\text { Percentage of relatives with } \\
\text { allergy manifestations }\end{array}$ & $95 \% \mathrm{CI}$ \\
\hline Mother & 393 & 98 & 24.94 & $20.25 \ldots .29 .62$ \\
\hline Father & 393 & 63 & 16.03 & $12.02 \ldots 20.05$ \\
\hline Maternal grandmother & 391 & 46 & 11.76 & $8.20 \ldots 15.33$ \\
\hline Paternal grandmother & 370 & 27 & 7.30 & $4.28 \ldots 10.32$ \\
\hline Maternal grandfather & 391 & 17 & 4.35 & $1.99 \ldots 6.70$ \\
\hline Paternal grandfather & 368 & 17 & 4.62 & $2.12 \ldots 7.12$ \\
\hline Maternal aunt & 155 & 17 & 10.97 & $5.22 \ldots 16.72$ \\
\hline Paternal aunt & 189 & 11 & 5.82 & $1.83 \ldots 9.81$ \\
\hline Maternal uncle & 185 & 19 & 10.27 & $5.19 \ldots 15.35$ \\
\hline Paternal uncle & 156 & 12 & 7.69 & $2.71 \ldots 12.67$ \\
\hline Sisters & 100 & 30 & 30.00 & $19.68 \ldots 40.32$ \\
\hline Female cousins & 156 & 19 & 12.18 & $6.21 \ldots .18 .15$ \\
\hline Brothers & 120 & 38 & 31.67 & $22.20 \ldots 41.13$ \\
\hline Male cousins & 167 & 28 & 16.77 & $10.29 \ldots .23 .24$ \\
\hline Total & 3534 & 442 & & \\
\hline
\end{tabular}

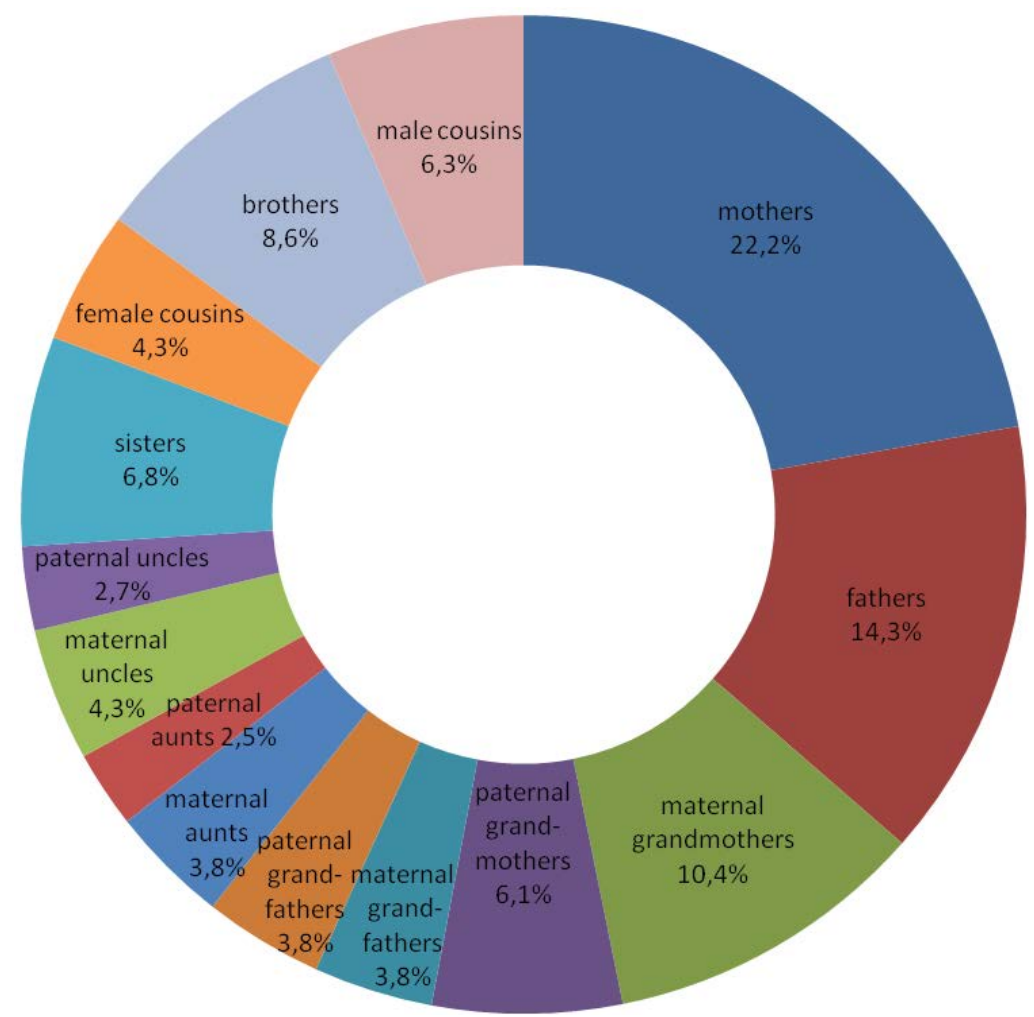

Figure 1. Relatives' kinships percentage in the total number of suspected allergy manifestations in families of newborns

The largest percentage of relatives' kinships in the total number of allergy manifestations (see Figure 1) is in parents of newborns. However, the percentage in the total number of allergies, even the largest one, is weak for the prediction. The prediction model based on parental atopy, maternal education and gender gives a low area under the 
receiver-operating characteristic curve - 0.63 (95\% CI $0.60-0.67)$, as shown by Wen et al [1]. The sensitivity of information on allergy manifestations in parents, as well as in other relatives, is also not really high, as shown by Treneva [5], hence is not suitable in a primary prevention allergy group setting. This purpose criterion for "a negative group" setting, which needs a further study, is possibly within the high specificity of information on absence of allergy manifestations in male relatives, which relates to the low rates of allergy manifestations in both mother/father grandfathers (see Table 1).

Our results show an increasing trend of allergy incidence with successive generations (see Figure 2). That's coincides well with the known worldwide trends of allergy incidence changes throughout the last few decades, as noted by Eder et al [7]. We show that allergy incidence increases from $7,04 \%$ in grandparents, to $14,96 \%$ in parents/aunts/uncles, to $21,18 \%$ in siblings and cousins. An increasing of allergy prevalence within a genetically stable population could be explained by environmental changes. Results of many studies worldwide lead to the same hypothesis. German Infant Nutritional Intervention (GINI) Study showed positive association between air pollution and sensitization to common allergens in preschool children, as noted by Brauer at al [8] and study done by Heinrich and Wichmann [9] found an association between air pollution and raised total IgE and sensitization to inhalant allergens in children.

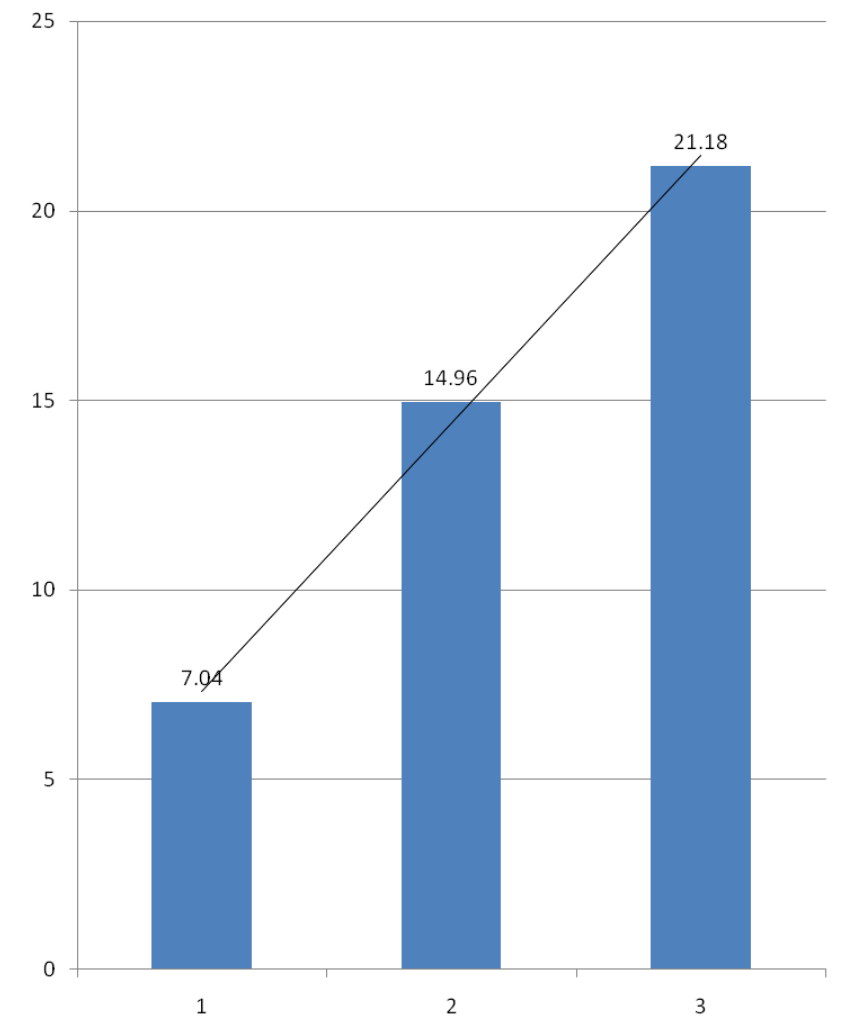

Figure 2. Suspected allergy manifestations prevalence (\%) in relatives' generations of Moscow newborns-2011 cohort

1 - grandparents' generation

2 - parents' generation

3 - siblings' generation

Using the frequencies (see Table 1), we can predict the likelihood of allergies among newborns-2011 cohort. A family history with allergy manifestations in both parents and one female/male sibling gives a population probability value for allergic manifestations in a newborn child 55,9\% / 56,9\% respectively. These prognostic frequencies are obtained within a model of unconnected events probability used by Treneva and Pampura [10]. Undoubtedly, genetic predisposition to allergy will increase the probability of allergic manifestations in a child. Thus in children admitted to the Allergy and Immunology Unit of Research and Clinical Institute for Pediatrics at the Pirogov Russian National Research Medical University with diagnosed allergic diseases, the frequencies discussed above were expected to be $94 \%-96 \%$ as summarized by Treneva and Pampura [10]. There are no related papers in PubMed to compare the frequency of allergic manifestations in a child with allergies in both parents and one sibling.

Our study had certain limitations. Relatives were not medically examined. To ask for medical records of a large range and number of newborns' family members (grandparents, uncles/aunts, cousins as well as other relatives) at the postnatal department where mothers of newborns provided us with the information was impossible and impracticable. Mothers' answers could contain incorrect information with some exaggerations as well.

At the same time dealing with all relatives' history alone even with unproved laboratory investigations may consider not only a limitation, but certain strength of the study. Allergy tests in general practice are aimed mainly to confirm IgE type of reactions. Other allergy types remain underestimated. Single transition reactions not registered by a doctor (food allergy, urticaria), single-pass mild drug allergy may be restricted in medical documentation. So history alone may expand the view in comparison with usually proved via laboratory investigations IgE type of allergy reactions.

\section{Conclusion}

There are clear gender differences with increased suspected allergic conditions diagnosed in female relatives of two preceding the newborn generations compared with male relatives of the same generations and a tendency to increased suspected allergic manifestations among women of maternal relatives in comparison to paternal relatives. Females are seemed to be more capacious to allergy. Grandfathers are seemed to be more rigid regardless to paternal or maternal family.

$12.51 \%$ of Moscow newborns-2011 cohort relatives have suspected allergic conditions with a trend of decreasing incidence with successive generations i.e. same generation (siblings/cousins) have greater allergic incidence than $(>)$ second generation (parents/aunts/uncles) $>$ first generation (grandparents).

\section{Acknowledgments}

We are grateful for Moscow No.1 maternity hospital executive chief Nickolay $Y$. Ivannykov, postnatal department chief Tatyana A. Shkersten, physician Larisa A. Lykhanova and all mothers interviewed for their cooperation. We would like to thank Dr. Annabella Procktor for her helpful assistance in manuscript preparation. We are grateful to Professor John O. Warner for his critical appraisal of our paper. 


\section{Abbreviations Used}

AD: Atopic dermatitis

RAD: Respiratory allergic diseases

OR: Odds ratio

GINI: German Infant Nutritional Intervention

\section{Declaration of All Sources of Funding}

The study was not funded. The Authors have no commercial associations that might pose a conflict of interest. None has received any money for this study.

\section{Authors' Contributions}

Dr.Treneva M. gave the idea of the paper, collected and interpreted clinical data. Dr. Pampura A. and Dr.Munblit D. gave a substantial contribution to manuscript design, revised it critically. All Authors contributed to the drafting of the manuscript and approved the final manuscript.

\section{References}

[1] Wen H-J, Wang Y-J, Lin Y-C, Chang C-C, Shieh C-C, Lung F-W et al. Prediction of atopic dermatitis in 2-yr-old children by cord blood IgE, genetic polymorphisms in cytokine genes, and maternal mentality during pregnancy. Pediatr Allergy Immunol 2011:22; 695-703.

[2] Kynyk JA, Mastronarde JG, McCallister JW. Asthma, the sex difference. Curr Opin Pulm Med 2011; 17(1):6-11.

[3] Lødrup Carlsen KC, Söderström L, Mowinckel P, Håland G, Pettersen M, Munthe Kaas et al. Asthma prediction in school children; the value of combined IgE-antibodies and obstructive airways disease severity score. Allergy 2010; 65(9):1134-40.

[4] Arshad SH, Karmaus W, Raza A, Kurukulaaratchy RJ, Matthews $\mathrm{SH}$, Holloway JW et al. The effect of parental allergy on childhood allergic diseases depends on the sex of the child. J Allergy Clin Immunol 2012; 130:427-34.

[5] Treneva MS. Allergic diseases primary prevention children setting positive predictive value and high specificity of information on allergic diseases in male relatives. Russian Allergy Journal 2010; 6:34-37.

[6] Kanny G, Moneret-Vautrin DA, Flabbee J, Beaudouin E, Morisset M, Thevenin F. Population study of food allergy in France. J Allergy Clin Immunol. 2001; 108(1):133-40.

[7] Eder W, Ege MJ, von Mutius E. The asthma epidemic. N Engl J Med 2006;355(21): 2226-35.

[8] Brauer M, Hoek G, Smit HA, de Jongste JC, Postma DS, Kerkhof $\mathrm{M}$ et al. Air pollution and development of asthma, allergy and infections in a birth cohort. Eur Respir J 2007; 29:879-88.

[9] Heinrich J, Wichmann HE. Traffic related pollutants in Europe and their effect on allergic disease. Curr Opin Allergy Clin Immunol 2004;4:341-8.

[10] Treneva MS, Pampura AN. Allergic disease probability in children with relatives' allergic manifestations. Russian Allergy Journal 2006; 3: 40-43. 